\title{
ON THE CORRECTION TO PRECESSION FROM PROPER MOTIONS REFERRED TO GALAXIES
}

\author{
S. VASILEVSKIS and A. R. KLEMOLA \\ Lick Observatory, University of California, Santa Cruz, Calif., U.S.A. \\ (Presented at IAU Colloquium No. 9, 'The IAU System of \\ Astronomical Constants', Heidelberg, Germany, August 12-14, 1970.)
}

One of the principal aims of the Lick proper motion program, as conceived and initiated by Wright (1950), was to derive the correction to precession. Ideally, proper motions of stars from a fundamental catalogue should be measured with respect to galaxies. Unfortunately, these stars are too bright for a direct reference to faint galaxies, even with an objective grating and two systems of exposures ( $2 \mathrm{~h}$ and $1 \mathrm{~min}$ ) on every plate, as employed at Lick. For this reason a cooperative project with the U.S. Naval Observatory was initiated in 1953 (Scott, 1954; Vasilevskis, 1954), with an intention to establish a direct relationship between meridian circle and Lick observations. When Heckmann (1954) proposed the formation of the AGK3, it was agreed to discontinue the cooperation mentioned, so as to make the resources of the U.S. Naval Observatory available for observing the AGK3 reference stars, and then to use the AGK3 as an intermediary for relating the Lick proper motions to a fundamental system. An obvious advantage of this change was offered by the abundance of AGK stars for measurement on Lick plates; a disadvantage is the absence of the AGK3 data south of declination -2.5 .

First-epoch Lick plates were taken with the 20-in. lens corrected for blue light; 1246 fields north of $-23^{\circ}$ were photographed during the period from 1947 to 1954 . A pilot program was started in 1968, with an intention to investigate observational errors, to develop appropriate programs and routines for reductions, and to derive some preliminary astronomical results, such as the correction to precession, data on solar motion and constants of galactic rotation. A total of 97 fields, nearly uniformly distributed over the sky north of $-23^{\circ}$ were photographed and measured, and stellar proper motions were derived in 78 regions located outside the zone of avoidance for galaxies. Photographs were taken not only with the original blue lens, but also with the new objective corrected for yellow light. Proper motions of the AGK3 stars selected for measurement at Lick were kindly communicated to us by Dr W. Dieckvoss. First preliminary results were reported at the IAU Colloquium No. 7 at Minneapolis, in April 1970 (Vasilevskis and Klemola, 1970), and will be referred to as Test I. The present report deals only with the correction to precession from a more detailed analysis of proper motions; it will be referred to as Test II. Results concerning stellar dynamics will be discussed elsewhere.

Results for the correction to precession, Test I, departed significantly from the recently derived values by others (e.g. Fricke, 1967), and it was assumed that the Lick proper motions might have been responsible for the discrepancy. There seemed to be 
several possible sources of systematic errors. First, both the blue and yellow secondepoch plates were measured, and then the measurements of yellow photographs were reduced to those of blue ones, and finally means of both sets of positions were taken. These means were compared with the first-epoch measurements of blue photographs only, for derivation of proper motions. It was conceivable that the reduction from yellow to blue plates could contain an undetected color effect. Second, images of the systems I and II, i.e. of long and short exposures, were lumped together without a proper investigation of possible differences between both systems. Finally, straight means of proper motion differences AGK3-Lick were formed for every Lick plate, and the equatorial coordinates of plate centers were used instead of mean coefficients used in Equations (3).

Results to be reported here, Test II, are derived only from plates taken in blue light. Since these sets of photographs are practically identical in their properties for both the epochs, no significant systematic effect can be expected. The faintest of the selected AGK3 stars were measured only in System I, the brightest ones in II, and those of intermediate brightness in both the systems. From 766 stars measured in both systems the differences System I-System II in $x$ and $y$, i.e. in $\Delta \alpha \cos \delta$ and $\delta$, were obtained as $+0.06 \pm 0.02$ and $-0.04 \pm 0.03$ per century, respectively. Although these differences are small, it was decided to carry out the reductions separately for each system of measurements. Regarding the combination of proper motions on each plate, several approaches were tried.

A total of 59 fields were used for the study reported here: almost all the remaining regions are either in the zone of avoidance, or south of -2.5 . A total number of 1898 AGK3 stars were measured, 898 of them in System I, 1768 in System II, and 766 in both of the systems. Since all stars on a Lick plate are systematically affected by the errors of the plate constants derived from the reference to galaxies and from the reduction of System II to System I, the $898+1768=2666$ proper motions obtained cannot be considered as independent. A kind of 'normal' proper motions differences AGK3-Lick must be obtained for each plate, therefore, and then the 59 regions can be treated as independent units.

At first, proper motion differences for each star in a particular region, covered by a Lick plate, were expressed separately in $x$ and $y$ as

$$
\Delta \mu=a+b x-c y+d m,
$$

where $x$ and $y$ are rectangular coordinates of a star, and $m$ is its magnitude. The constants $a, b, c$ and $d$ were then found from least squares solutions. The first constant, $a$, was then considered as the 'normal' proper motion difference for the plate center, while the remaining constants were supposed to take care of the scale error, orientation error including that caused by the error of precession, and of a possible magnitude effect. The latter seemed to be present, because in general the faintest stars gave the largest differences. Further analysis, however, showed that in most cases the value obtained for the constant $d$ was spurious, caused by a few abnormally large proper motion differences. Further study revealed that there is a pronounced correlation 
between these differences and the AGK3 proper motions. Five regions with the highest numbers of large differences were selected, and all the differences in $x, \Delta \mu_{x}$, exceeding 2 "/century are plotted in Figure 1 versus AGK3 proper motions $\mu_{x}$. About $30 \%$ of the AGK3 proper motions leading to the largest differences were designated as doubtful by Dr Dieckvoss; Figure 1 shows that more spurious proper motions are expected to be present in the AGK3, and unless most of them are not removed in the present investigation, spurious results for correction to precession can be expected.

Eight solutions, each in System I and II separately were performed, with cutoffs of proper motion differences ranging from $9^{\prime \prime}$ to $2^{\prime \prime}$ per century; the percentage of stars rejected at various cutoffs is shown in Figure 2, where open circles represent System I and the filled ones are for System II. Within reasonable limits, the probability of a genuine spurious error should not depend on its size, hence the percentage of rejected stars should increase linearly with the decrease of cutoff. The percentage of normally distributed observational errors, however, should not exhibit this linear trend. Figure 2 shows that a reasonable limit for rejection of spurious data might be around 6 ".

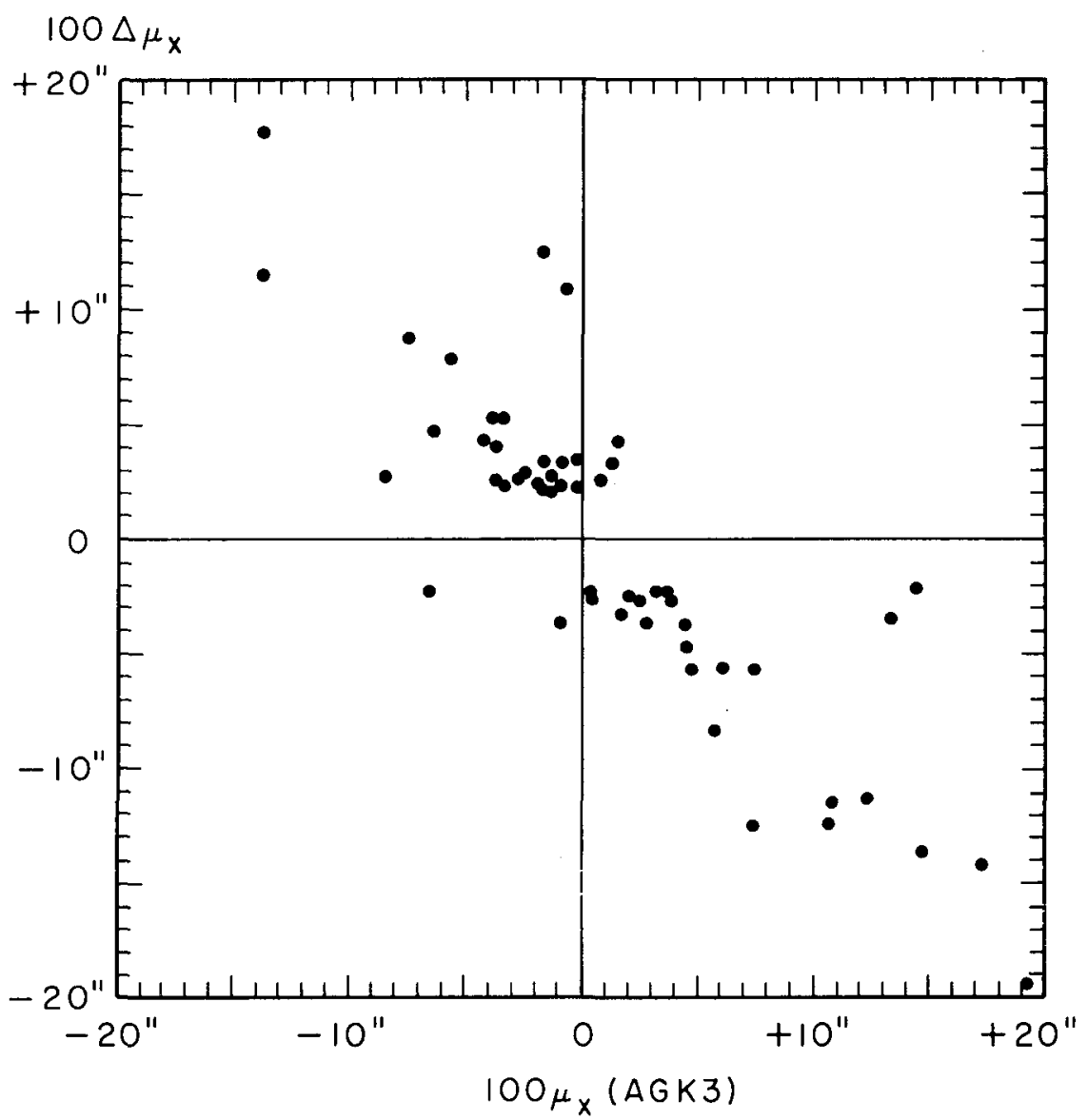

Fig. 1. Relationship between proper motion differences and AGK3 proper motions. 


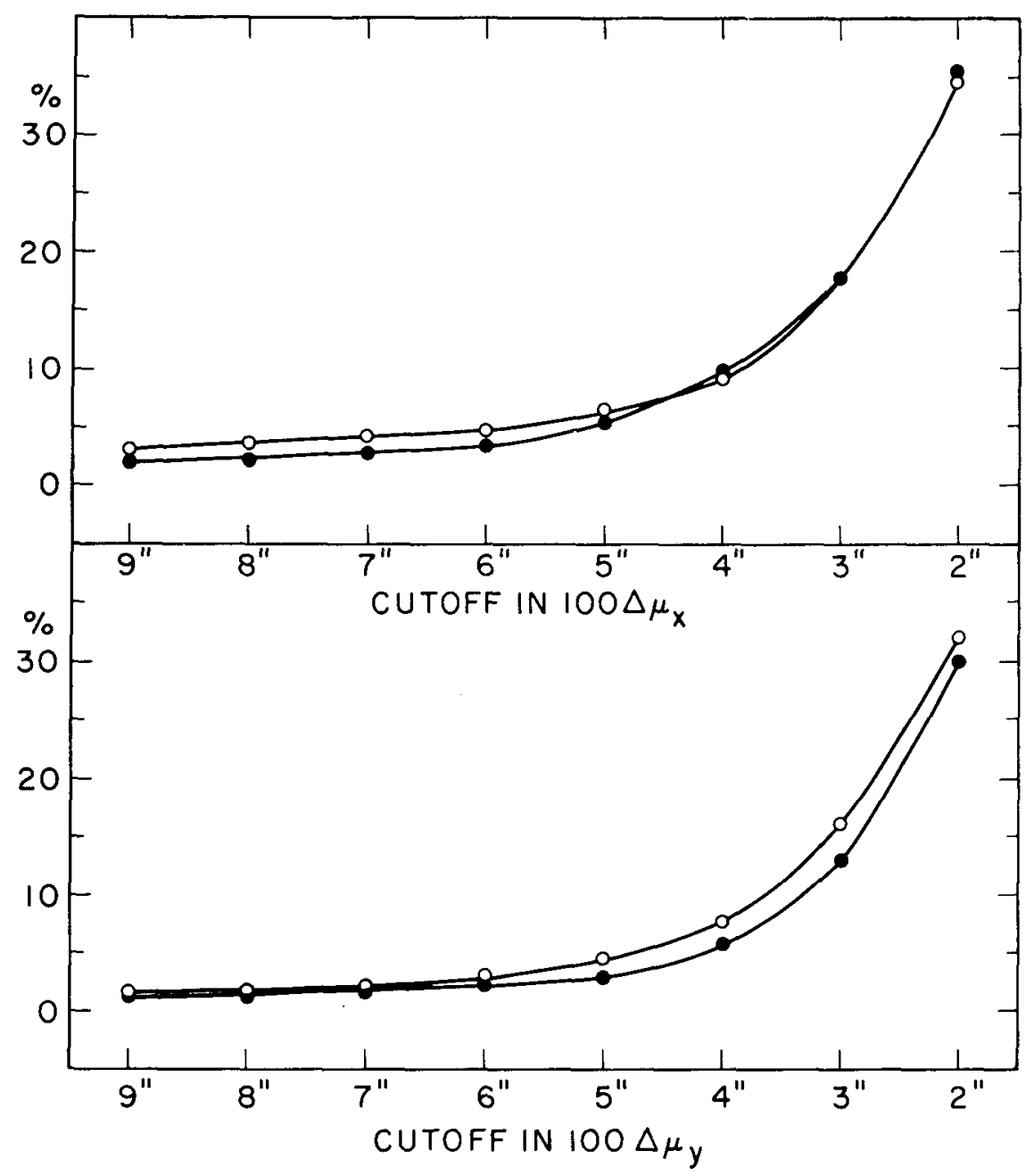

Fig. 2. Percentage of rejected measurements.

After various trials of combining proper motion differences in individual regions, the finally adopted procedure was as follows. Since proper motions of stars are eliminated from the differences, no terms for solar motion and galactic rotation need to be taken into account and the effect of precession errors can be computed from:

$$
\begin{aligned}
\Delta n \sin \alpha \sin \delta+\Delta k \cos \delta & =\Delta \mu_{x} \\
\Delta n \cos \alpha & =\Delta \mu_{y}
\end{aligned}
$$

Means for all the stars on a plate were formed, leading to

$$
\begin{aligned}
\Delta n \overline{\sin \alpha \sin \delta}+\Delta k \overline{\cos \delta} & =\overline{\Delta \mu_{x}} \\
\Delta n \overline{\cos \alpha} & =\overline{\Delta \mu_{y}}
\end{aligned}
$$


or, written in a simplified form:

$$
\begin{aligned}
\Delta n A+\Delta k B & =\overline{\Delta \mu_{x}} \\
\Delta n C & =\overline{\Delta \mu_{y}}
\end{aligned}
$$

For each plate the mean proper motion differences and the coefficients $A, B$ and $C$ were computed, for each system and cutoff mentioned before, and thus 59 pairs of weighted Equations (3) were formed for the derivation of $\Delta n$ and $\Delta k$ in every case. Similar solutions were also repeated with added terms for correction to the secular variation of obliquity, $\Delta D$, recently discussed by Aoki (1967) and Lieske (1970); the corresponding terms are $-\Delta D \cos \alpha \sin \delta$ and $\Delta D \sin \alpha$ in $x$ and $y$, respectively. It may be

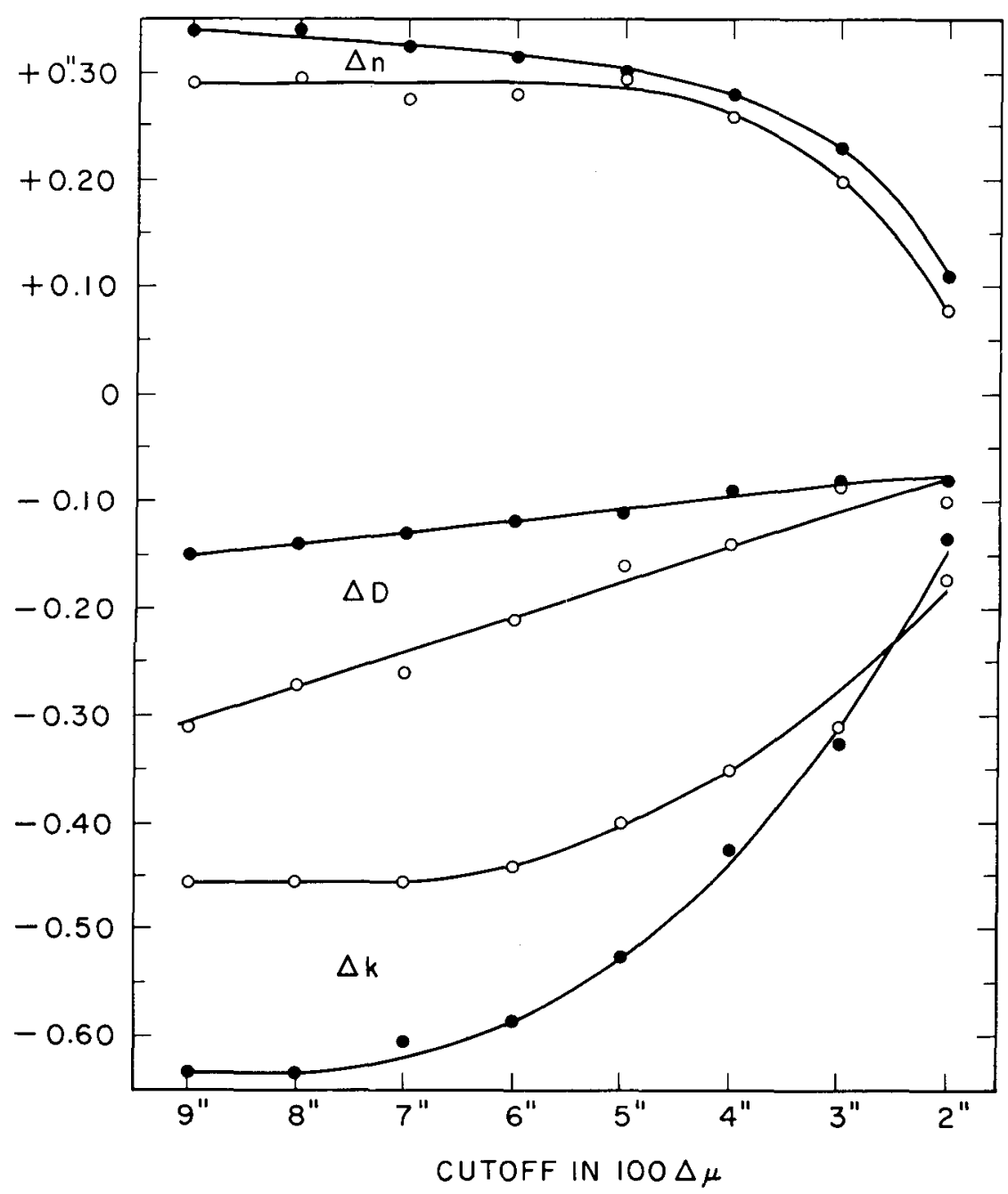

Fig. 3. Correction $\Delta n, \Delta k, \Delta D$. 
noted that since the proper motion differences are not affected by the constant $B$ of galactic rotation, they offer an opportunity to derive $\Delta D$ separately from $B$.

Solutions of Equations (3) were performed in $x, y$ and combined, for both systems of images and for all the cutoffs mentioned earlier, without and with the terms for $\Delta D$, thus bringing the total number of separate solutions to 108 . The results from combined solutions are shown in Figure 3; open circles represent System I and the filled ones are for System II. Since the values of $\Delta n$ and $\Delta k$ are practically the same whether the terms for $\Delta D$ are omitted or included, these two cases are not shown separately. As can be seen, $\Delta n$ is practically constant down to the cutoff of $5^{\prime \prime}$, and there is no large discrepancy between the System I and II. This does not apply to $\Delta k$, the absolute value of which starts to decrease already at $7^{\prime \prime}$, and there is an appreciable discrepancy between the System I and II. The $\Delta D$ changes almost linearly with the cutoff in the whole region covered by Figure 3.

TABLE I

Correction to precession from proper motion differences smaller than $6^{\prime \prime} /$ century

\begin{tabular}{|c|c|c|c|c|c|c|c|c|}
\hline System & $\Delta D$ & $\begin{array}{l}\text { Proper } \\
\text { motions }\end{array}$ & $\Delta n$ & & $\Delta k$ & & $\Delta D$ & \\
\hline \multirow[t]{2}{*}{ I } & \multirow[t]{4}{*}{ excluded } & in $x$ & \multicolumn{2}{|c|}{$+0.38 \pm 0^{\prime \prime} 12$} & \multicolumn{2}{|c|}{$-0 " 45 \pm 0.12$} & & \\
\hline & & in $y$ & 26 & 13 & & & & \\
\hline 853 p.m.'s in $x$ & & combined & 28 & 12 & 45 & 12 & & \\
\hline \multicolumn{8}{|l|}{$869 \quad y$} & \\
\hline \multirow[t]{3}{*}{$\bar{m} p g=11.0$} & included & in $x$ & 36 & 26 & 40 & 12 & -0.58 & 0.27 \\
\hline & & in $y$ & 26 & 13 & & & 10 & 16 \\
\hline & & combined & 28 & 12 & 43 & 12 & 21 & 14 \\
\hline \multirow[t]{2}{*}{ II } & excluded & in $x$ & 38 & 20 & 59 & 9 & & \\
\hline & & in $y$ & 30 & 13 & & & & \\
\hline \multicolumn{2}{|l|}{1699 p.m.'s in $x$} & combined & 32 & 10 & 59 & 11 & & \\
\hline 1719 & & & & & & & & \\
\hline \multirow[t]{3}{*}{$\bar{m} p g=10.1$} & included & in $x$ & 38 & 20 & 57 & 9 & 25 & 21 \\
\hline & & in $y$ & 30 & 13 & & & 9 & 15 \\
\hline & & combined & 32 & 10 & 58 & 11 & 12 & 12 \\
\hline
\end{tabular}

Results for the cutoff of $6^{\prime \prime}$ are given in Table I, and they offer an additional insight into peculiarities of the solutions. As mentioned, neither $\Delta n$ nor $\Delta k$ depend on omitting or including the terms for the correction to secular variation of obliquity. There is a reasonable agreement between the values of $\Delta n$ obtained from proper motions in $x$ and $y$, and in System I and II. The reality of $\Delta D$ is not supported by this study; the discrepancies between solutions in $x$ and $y$ are so large as to indicate that the values obtained might have been caused by periodic errors, perhaps mainly $\Delta \alpha_{\alpha}$ and $\Delta \delta_{\alpha}$ in proper motions. The values obtained for $\Delta k$ are up to almost three times as large as the most probable value adopted by Fricke (1967). It does not mean, of course, that $\Delta k$ obtained from proper motions and from their differences must necessarily agree. Proper motions require a simultaneous solution for $\Delta k$ and the constant $B$ of galactic 
rotation, while proper motion differences do not involve $B$. Consequently, possible systematic errors of the catalogues may have different effect in each case. The large discrepancy between $\Delta k$ from System I and II in Table I and Figure 3 shows, however, that errors of proper motions in $x$ must be held primarily responsible for the departure from a more reasonable value. The numbers of stars in Table I show that more large differences occur in $x$ than in $y$; the same is supported by inspection of results in all the 59 fields. Moreover, Figure 1 indicates that there is still a strong correlation between the differences and proper motions below the cutoff of 6 ", represented in Table I. Figure 3 indicates, perhaps accidentally, that $\Delta k$ in both systems assumes the same value at a cutoff of 2.5 , and this value is close to that adopted by Fricke. Since stars measured in System II are one magnitude brighter than those in System I, a magnitude effect may be at least partly responsible for the discrepancy.

As the discussion above indicates, no attempt would be justified to single out certain of the derived values as the most probable for $\Delta n$, and particularly $\Delta k$; regarding $\Delta D$, its significance seems doubtful. Since less than $5 \%$ of all the Lick plates available for proper motions have been used for derivation of the data presented here, great improvement is possible in the course of expansion of the present work, if certain criteria are met.

The discussion of stellar proper motions referred to galaxies can be made in two separate directions. A comparison with proper motions referred to a fundamental system, e.g. FK4, yields differences that consist solely of correction to precession and other systematic errors that affect both types of proper motions in a different way, and these differences do not depend on proper motions. On the other hand, since proper motions with respect to galaxies do not contain the errors of reference to the equator and equinox, they are uniquely suited for investigation of systematic motions of stars, including the sun. Moreover, our experience shows that the highest precision from the Lick plates is for stars between photographic magnitudes 10 and 16, and this fact enhances the strength of the Lick program in problems of stellar dynamics.

The prospect of reliable determination of correction to precession will depend primarily on the strength of connection between proper motions in a fundamental system and those with respect to galaxies, and of precision of both types of proper motions. We were disappointed to find out that the precision of Lick measurements of bright stars starts to decrease earlier than anticipated; around photographic mag. 9.5 this decrease becomes already noticeable, and measurements of stars brighter than magnitude 8 cannot be considered as reliable. For this reason only the fainter AGK3 stars were chosen for comparison; the mean magnitude of these stars is given in Table I. As was indicated before, the reliability of the AGK3 proper motions seems to decrease with the brightness of stars, and consequently, there is no sufficiently strong overlap between AGK3 and Lick. Unless this overlap is extended and strengthened, and in addition, unless proper motions of a sufficient number of faint stars in a fundamental system become available south of -2.5 , no immediate great contribution can be expected from the Lick program in determination of the correction to precession. In that case our efforts will need to be directed mainly towards the area of the principal 
strength of the program, i.e. to problems of stellar dynamics. Hopefully, our results on solar motion and galactic rotation may contribute indirectly to studies of precession from proper motions in a fundamental system.

The above conclusion does not necessarily apply to a more distant future, say after two decades, when repetition of the Lick photographs in yellow light will be justified. Because of much smaller and better images, and the use of a different objective grating, as compared with the blue lens, it will be possible to derive reliable proper motions for much brighter stars than now, hopefully at least to photovisual mag. 7. This extension in magnitude is expected to permit a strong overlap with catalogues in a fundamental system, thus leading to reliable direct determination of the correction to precession.

We are greatly indebted to Dr W. Dieckvoss for making the AGK3 proper motions available before their publication, and to $\mathrm{Mr} \mathrm{C}$. A. Wirtanen for his major contribution in survey and measurement of plates. The project has been supported in great part by the National Science Foundation.

\section{References}

Aoki, S.: 1967, Publ. Astron. Soc. Japan 19, 585.

Fricke, W.: 1967, Astron. J. 72, 1368.

Heckmann, O.: 1954, Astron. J. 59, 31

Lieske, J. H.: 1970, Astron. Astrophys. 5, 90.

Scott, F. P.: 1954, Astron. J. 59, 44.

Vasilevskis, S.: 1954, Astron. J. 59, 40.

Vasilevskis, S. and Klemola, A. R.: 1970, in W. J. Luyten (ed.), 'Proper Motions', IAU Coll. No. 7, University of Minnesota, Minneapolis, p. 167.

Wright, W. H.: 1950, Proc. Am. Phil. Soc. 94, 1. 